\title{
New Lessons from the First Galactic Stars
}

\author{
J. Andersen ${ }^{1,2}$ and B. Nordström ${ }^{1}$ \\ ${ }^{1}$ Niels Bohr Institute, Copenhagen University, Denmark \\ email: ja@astro.ku.dk, birgitta@astro.ku.dk \\ ${ }^{2}$ Nordic Optical Telescope, Santa Cruz de La Palma, Spain
}

Abstract. Main results from a study of extremely metal-poor stars are briefly summarised.

Keywords. cosmology: early universe, galaxies: evolution, stars: abundances

\section{Introduction}

Our best guides to the structure of the first generation of stars in the Galaxy and the heavy elements produced during their evolution are the most metal-poor stars in the Galaxy. The "First Stars project (Cayrel et al. 2004) aims to analyse a sample of 75 extremely metal-poor $(\mathrm{EMP})$ halo stars $([\mathrm{Fe} / \mathrm{H}]<-2.5)$ as accurately and homogeneously as possible, using high-resolution, high-S/N spectra from the ESO VLT and the UVES spectrograph (Cayrel et al. 2004, François et al. 2007). Here we briefly summarise a few of the most salient results on behalf of the "First Stars team": M. Spite, P. Bonifacio, R. Cayrel, F. Spite, V. Hill, J. Andersen, B. Barbuy, T. C. Beers, E. Depagne, P. François, P. Molaro, B. Nordström, B. Plez and F. Primas.

\section{Li Abundances and the Spite Plateau}

The Li abundance of the most metal-poor stars, the Spite Plateau, is believed to reflect the $\mathrm{Li}$ abundance generated in the Big Bang, and is an important observational check on BBNS models. We have added new, accurate measurements for 19 EMP dwarfs and find a mean level of the plateau 0.3 dex below that of standard BBNS models constrained by the WMAP data (Bonifacio et al. 2007). While this may be due to processes such as diffusion (Korn et al. 2006), uncertainties in the temperature scale remain important.

\section{The r-process and the Actinides}

In EMP stars the elements beyond the Fe peak are formed by fast neutron capture, the r-process. The $[\mathrm{r} / \mathrm{Fe}]$ ratios range from below solar to 50 or more in extreme, very rare EMP giants, which show the entire range of r-elements up to Th and U. The relement ratios in EMP giants, presumably enriched by very few SN events, are remarkably consistent with the r-process pattern of the Solar system, accumulated over many stellar generations. However, the production of Th and U seems decoupled from the rest of the actinides in CS31082-001 (Hill et al. 2001, Cayrel et al. 2001), but not in other known cases e.g. HE1523-0901 (Frebel et al. 2007). The Th and U lines in CS31082-001 also made it possible to derive an age of that star by radioactive age-dating. The r-process enhancement appears unrelated to the $\mathrm{C}$ enhancement affecting some EMP giants. 


\section{Early Mixing of the ISM}

An important question in early galactic evolution is the efficiency of mixing of the ISM, as reflected in the scatter of abundance ratios of EMP stars. Due to our high-quality spectra and homogeneous analysis, the observational scatter of our abundance ratios is very much lower than in previous studies, and suggests that mixing of the primitive ISM was very efficient (Cayrel et al. 2004). However, some real outliers are found e.g. CS22949-37 (Depagne et al. 2002).

A crucial assumption when deriving the composition of the progenitor(s) of the EMP stars is that the stellar surface reflects the composition of the interior. This appears to be the case for most elements, but there are prominent exceptions, notably $\mathrm{C}$ and $\mathrm{N}$ in EMP giants (Spite et al. 2006). We find that the most-evolved half of the stars show products of $\mathrm{CN}$ cycling mixed to the surface, with the N-rich stars having become $\mathrm{C}$ poor and the $\mathrm{Li}$ abundance and ${ }^{12} \mathrm{C} /{ }^{13} \mathrm{C}$ ratios dropping at the same time. In the most extreme cases, $\mathrm{Na}$ and $\mathrm{Al}$ are enhanced as well (Cayrel et al. 2004). O is not significantly affected, however, limiting the depth of the extra mixing.

\section{Conclusions}

Much can still be learned on the early Galactic evolution from high-quality spectroscopy of EMP stars. Further results from the "First Stars" will be reported in future papers.

\section{References}

Bonifacio, P., Bonifacio, P., Molaro, P., Sivarani, T., Cayrel, R., Spite, M., Spite, F., Plez, B., Andersen, J., Barbuy, B., Beers, T.C., Depagne, E., Hill, V., François, P., Nordström, B., \& Primas, F., 2007, A\&A 462, 851(Paper VII)

Cayrel, R., Hill, V., Beers, T.C., Barbuy, B., Spite, M., Spite, F., Plez, B., Andersen, J., Bonifacio, P., François, P., Molaro, P., Nordström, B., \& Primas, F., 2001, Nature 409, 692

Cayrel, R., Depagne, E., Spite, M., Hill, V., Spite, F., François, P., Plez, B., Beers, T.C., Primas, F., Andersen, J., Barbuy, B., Bonifacio, P., Molaro, P., \& Nordström B., 2004, A\&A 416, 1117 (Paper V) (astro-ph/0311082)

Depagne, E., Hill, V., Spite, M., Spite, F., Plez, B., Beers, T.C., Barbuy, B., Cayrel, R., Andersen, J., Bonifacio, P., François, P., Nordström, B., \& Primas, F., 2002, A\&A 390, 187 (Paper II) Spite, F., Plez, B., Bonifacio, P., 1105

François, P., Spite, M., Bonifacio, P., Cayrel, R., Hill, V., Spite, F., Plez, B., Depagne, E., Barbuy, B., Molaro, P., Beers, T. C Andersen, J., Nordström, B., \& Primas, F., 2007, A\&A in prep. (Paper VIII)

Frebel, A., Johnson, J.L., \& Bromm, V., 2007, ApJL subm. (astro-ph/0701395)

Hill, V., Plez, B., Cayrel, R., Beers, T.C., Nordström, B., Andersen, J., Spite, M., Spite, F., Barbuy, B., Bonifacio, P., Depagne, E., François, P., Molaro, P., \& Primas, F., 2002, A\&A 387, 560 (Paper I)

Korn, A.J., Grundahl, F., Richard, O., Barklem, P.S., Mashonkina, L., Collet, R., Piskunov, N., \& Gustafsson, B., 2006, Nature 442, 657 (astro-ph/0608201) (Paper IV)

Spite, M., Cayrel, R., Hill, V., Spite, F., François, P., Plez, B., Bonifacio, P., Molaro, P., Depagne, E., Andersen, J., Barbuy, B., Beers, T.C., Nordström, B., \& Primas, F., 2006, A\&A 455, 291 (Paper IX)(astro-ph/0605056)

P., Cayrel, R., Herwig, F., Barbuy, B., Depagne, E., \& Hill, F., 2006, A\&A 459,125 